\title{
Aspectos psicossociais em desastres socioambientais de origem geoclimática: uma revisão integrativa da literatura
}

\author{
Psychosocial aspects in socioenvironmental disasters of geoclimatic \\ origin: an integrative literature review
}

Milena Maciel de Carvalho', Simone Santos Oliveira ${ }^{\mathbf{1}}$

DOI: $10.1590 / 0103-11042020 E 223$

RESUMO Dentre os inúmeros atravessamentos que permeiam a conjuntura de um desastre, os aspectos psicossociais merecem destaque por incluírem questões culturais, sociais, estruturais, psíquicas, sanitárias e simbólicas. O objetivo desta revisão foi apresentar como esses aspectos têm sido explorados na literatura científica nos últimos 20 anos (1997-2016) por autores e periódicos de países da América do Sul, em contextos de desastre socioambiental de origem geoclimática. Foi realizado levantamento bibliográfico nas bases eletrônicas Capes, SciELO, Latindex e Portal da Biblioteca Virtual em Saúde. Levando-se em conta os critérios de inclusão e exclusão adotados nesta revisão, 52 publicações constituíram a amostra final. O levantamento revelou a predominância das temáticas 'saúde mental', 'impactos psicossociais e na saúde', 'saúde pública' e 'gestão de riscos'. Os resultados evidenciaram a desigualdade social como fator potencializador de desastres e a ineficiência de políticas públicas no Brasil para a redução de riscos de desastres, bem como o predomínio de estudos que associam o apoio psicossocial a intervenções restritas ao campo da saúde mental. Como caso oposto, o artigo apresenta a construção da identidade e das políticas públicas chilenas em função dos terremotos ocorridos no País.

PALAVRAS-CHAVE Desastres. Mudança climática. Impacto psicossocial. Sistemas de apoio psicossocial. América do Sul.

ABSTRACT Among the many crossings that permeate the conjuncture of a disaster, the psychosocial aspects deserve highlighting, because they include the cultural, social, structural, psychic, health, and symbolic issues. The aim of this review is to present how these aspects have been explored in the scientific literature in the last 20 years (1997-2016) by authors and journals from South American countries, in contexts of socioenvironmental disaster of geoclimatic origin. A bibliographic survey was conducted in the electronic databases Capes, SciELO, Latindex, and Virtual Health Library Portal. Taking into account the inclusion and exclusion criteria adopted in this review, 52 publications constituted the final sample. The survey revealed the predominance of 'mental health', 'psychosocial and health impacts', 'public health' and 'risk management' themes. The results showed evidence of social inequality as a potentiating factor of disasters and the inefficiency of public policies in Brazil in reducing disaster risks. Also, they revealed the predominance of studies that associate psychosocial support with interventions restricted to the field of mental health. As an opposite case, the article presents the construction of Chilean identity and public policies due to the earthquakes that occurred in the country.

1 Fundação Oswaldo Cruz (Fiocruz) - Rio de Janeiro (RJ), Brasil.

milenafiocruz@gmail.com
KEYWORDS Disasters. Climate change. Psychosocial impact. Psychosocial support systems. South America. 


\section{Introdução}

A população mundial tem acompanhado, particularmente nos últimos 20 anos, o aumento no número de pessoas afetadas por desastres socioambientais. Esse cenário traduz a conjuntura sociopolítica e econômica contemporânea do planeta, somada às questões ambientais e educacionais. Essa afirmação se fundamenta em dados dos principais órgãos de assistência e desenvolvimento mundiais, a exemplo da Organização das Nações Unidas (ONU) ${ }^{\mathbf{1 - 3}}$, do Banco Mundial ${ }^{4}$ e da Organização Mundial da Saúde $(\mathrm{OMS})^{5}$, assim como nas informações de órgãos de referência em estudos sobre mudanças climáticas ${ }^{6,7}$ e na produção científica internacional8-11.

Observa-se que a vulnerabilidade social é um elemento presente nos locais atingidos por fenômenos de origem natural que culminaram em desastres. Portanto, as questões sociopolíticas e econômicas têm um papel determinante no impacto de eventos extremos, visto que quase todos os países afetados por desastres são subdesenvolvidos ${ }^{\text {1-4,7-9. }}$.

Paralelamente, há um grande debate atual no que tange ao aumento da frequência e magnitude de eventos climáticos extremos. De acordo com o relatório 'The Global Climate 20112015’ da Organização Meteorológica Mundial (OMM), publicado na XXII Conferência Anual do Clima (COP22) realizada em Marrocos em 2016, houve, de fato, um aumento na frequência dos eventos meteorológicos extremos no planeta. Esse documento destaca a seca e as ondas de calor elevado como eventos extremos, que chamaram a atenção devido ao aumento de sua frequência ${ }^{12}$, e faz uma revisão de todos os eventos extremos reunidos no Boletim da Sociedade Americana de Meteorologia, ocorridos de 2011 a 2015, período que se mostrou peculiar historicamente. São mencionados como eventos emblemáticos nesse período: o derretimento de gelo no mar Ártico, a seca extrema que casou a morte de milhares de pessoas no Chifre da África, o furacão Sandy em 2012, o tufão Haiyan (Yolanda) em 2013 nas Filipinas, ondas de calor na Argentina e na Austrália e enchentes na Ásia. Quanto ao Brasil, o relatório faz menção à análise de três eventos extremos: as inundações e deslizamentos de terra na Região Serrana do estado do Rio de Janeiro em 2011, a forte seca entre os anos de 2013 e 2014, responsável pela crise hídrica em São Paulo, e a estiagem na Amazônia nos anos de 2014 e $2015^{12}$.

Mata-Lima et al. ${ }^{\mathbf{1 0}}$ indicam que os estudos realizados nos últimos 20 anos apresentam consistência na demonstração e previsão do aumento tanto da frequência como da intensidade de desastres socioambientais, especialmente os ligados ao clima. Além disso, mostram a relação entre esse tipo de desastre e o nível socioeconômico dos países afetados.

Um ponto convergente nas discussões sobre essa temática é a necessidade de as nações atuarem de forma planejada, estruturada e educativa na prevenção e resposta aos impactos dos fenômenos climáticos ${ }^{2,6,10}$. Essa postura preventiva se impõe não somente diante dos desastres relacionados com o clima, mas a todos os eventos extremos que ponham em risco a rotina e a vida das coletividades e excedam sua capacidade de resposta.

Esses e outros debates têm propiciado a expansão de estudos sobre o processo de intervenção em situações de desastre e emergência, possibilitando a otimização de práticas e o reconhecimento da importância dos diversos setores sociais, dos campos disciplinares e da própria comunidade no planejamento de ações nesses episódios. Nesse sentido, considerando-se a importância dessa articulação conjunta e de intervenções pautadas na prevenção de danos, as questões relativas ao impacto e ao suporte psicossocial nos desastres configuram-se como uma estratégia fundamental de prevenção, resposta e recuperação.

Segundo a própria constituição do termo, a perspectiva psicossocial pressupõe a combinação das relações que o indivíduo mantém com a sociedade e com o desenvolvimento do seu psiquismo; abarca questões que vão desde o suporte emocional, a saúde física e 
mental dos afetados até o apoio social, que inclui aspectos de suporte material, sanitário, espiritual e simbólico ${ }^{13}$. Portanto, para além da conjuntura relacionada com a mudança climática, compreender e ampliar as discussões sobre os aspectos psicossociais no contexto de desastre é fundamental, visto que as consequências psicossociais decorrentes de um desastre extrapolam as lesões físicas e emocionais e das perdas materiais e humanas. Contudo, as ações com essa finalidade ficam à margem da atenção por conta da ruptura violenta da rotina que um desastre acarreta.

Ao analisar os países mais afetados por desastres climáticos nos últimos 20 anos, vê-se que os países do continente americano não são os que mais sofrem esses impactos. No entanto, o Brasil aparece na lista dos 10 países mais afetados por desastres socioambientais em números absolutos (51 milhões de pessoas) ${ }^{2}$. Esse dado é mencionado no relatório 'The Human Cost of WeatherRelated Disasters 1995-2015', publicado em 2015 pelo Escritório das Nações Unidas para a Redução de Desastres (UNISDR) e Centro de Pesquisas de Epidemiologia em Desastres (Cred). A publicação baseou-se no banco de dados de eventos de emergência do Cred, o Emergency Events Database (EM-DAT), que reúne informações globais sobre a ocorrência de desastres relacionados com eventos naturais e tecnológicos e seus efeitos, desde $1900^{2}$.

Diante da conjuntura apresentada, torna-se imperativo discutir os aspectos psicossociais nesses cenários. Trazendo a discussão para o contexto da América do Sul e considerando as diferentes realidades sociopolíticas e econômicas desses países, o objetivo desta revisão é responder à seguinte pergunta: como os aspectos psicossociais têm sido explorados na literatura científica nos últimos 20 anos (1997-2016) por autores e periódicos de países da América do Sul, em contextos de desastre socioambiental de origem geoclimática?

Desse modo, pretende contribuir para o conhecimento científico acerca dos desastres ao apresentar o cenário contextual e prático dessa parte do globo e suas estratégias para minimizar os impactos sobre a saúde das populações nesses eventos.

\section{Desastres socioambientais de origem geoclimática}

É importante esclarecer o que está sendo chamado neste artigo de 'desastres socioambientais de origem geoclimática'. Primeiro, partiu-se da visão de que um fenômeno físico de origem ambiental (climatológico, geológico, hidrológico, meteorológico ou biológico) só se constitui em desastre quando um ambiente vulnerável, em interação com esse fenômeno, não tem capacidade de resposta, resultando em consequências negativas. Desse modo, tem de haver, necessariamente, a combinação entre uma ameaça, condições vulneráveis e capacidade insuficiente para responder a esse evento ${ }^{10,14,15}$.

O segundo aspecto é a natureza essencialmente social do desastre. Sem dúvida, todo desastre é social, uma vez que reflete as condições socioeconômicas, as formas de organização, a degradação dos ecossistemas do ambiente atingido, além de ser resultado da mudança climática em função do modo como a sociedade tem- se construído ${ }^{14-18}$. Portanto, entende-se a classificação dos desastres como sendo de origem socioambiental (ligada a eventos naturais) ou tecnológica (causada diretamente pelo homem, como rompimento de barragens, uso inadequado de substâncias tóxicas e nucleares etc.). Neste artigo, o foco são os desastres socioambientais (oriundos de eventos naturais) relativos exclusivamente ao clima e a aspectos geofísicos, ou seja, os geoclimáticos.

De forma geral, os desastres desencadeados por fenômenos 'naturais' são classificados como de origem climatológica (seca, estiagem, incêndios florestais), hidrológica (inundações, enxurradas e alagamentos), meteorológica (ciclones, tornados, ondas de calor), geológica ou geofísica (deslizamentos, 
erosão e terremotos) ou biológica (epidemias, infestações e pragas) ${ }^{\mathbf{1 9}}$.

A opção por incluir os eventos geofísicos na análise desta revisão levou em conta a relação entre esse tipo de evento e as mudanças climáticas, haja vista que estudos têm identificado a associação entre o aquecimento global e riscos geológicos, como terremotos e erupções vulcânicas, mostrando a influência das altas temperaturas do planeta e da consequente pressão sobre a crosta terrestre na ocorrência de deslocamento de placas tectônicas ${ }^{\mathbf{6}, 20-23}$.

Como delimitação hermenêutico-epistemológica, optou-se por qualificar como 'desastres geoclimáticos' todos os de origem climatológica, hidrológica, meteorológica e geológica, não incluindo os de origem biológica, como as epidemias por exemplo.

\section{Material e métodos}

Trata-se de um estudo de revisão integrativa, realizado por meio de levantamento bibliográfico em bases de dados indexadas. O levantamento ocorreu no ano de 2017, em que foram escolhidas as seguintes bases eletrônicas: Coordenação de Aperfeiçoamento de Pessoal de Nível Superior (Capes), Scientific Electronic Library Online (SciELO) e Sistema Regional de Información en Línea para Revistas Científicas de América Latina, el Caribe, España y Portugal (Latindex), além do Portal Regional da Biblioteca Virtual em Saúde (BVS). A BVS foi escolhida por concentrar informações técnico-científicas sobre saúde na América Latina e no Caribe, por congregar o centro especializado da Organização PanAmericana da Saúde (Opas)/OMS de informações científicas em saúde - a Bireme (Centro Latino-Americano e do Caribe de Informação em Ciências da Saúde) - e por incluir as bases Literatura Latino-Americana e do Caribe em Ciências da Saúde (Lilacs), Medline e uma especializada em desastres.

Inicialmente, adotaram-se como critérios de inclusão (fase 1 da busca bibliográfica) os seguintes tipos de publicação: artigos científicos, dissertações, teses e manuais que abordassem aspectos psicossociais em desastres nas Américas, no período de janeiro de 1997 a dezembro de 2016. As razões dessa busca mais genérica nesse momento inicial serão explicadas ainda neste tópico. Outro critério de inclusão adotado foi o de que os materiais estivessem nos idiomas português, inglês e/ou espanhol e publicados no período supracitado. Elegeram-se como critérios de exclusão textos com foco na saúde animal em desastres (medicina veterinária, zootecnia etc.) e publicações cujos resumos não estivessem disponíveis para acesso.

Foram utilizados descritores listados no DeCS (Descritores em Ciências da Saúde) que compreendessem os aspectos relacionados com os impactos, as intervenções e o apoio psicossociais nos desastres, tendo sido realizada pesquisa no DeCS para levantamento de todos os termos utilizados nas bases que explorassem essas três dimensões. Os seguintes descritores foram utilizados: 'desastres', 'impacto psicossocial', 'apoio psicossocial', 'apoio social', 'saúde mental', 'capacidade de resposta ante emergência', 'efeitos de desastre na saúde', 'planejamento em desastres', 'socorro em desastres', 'Resgate, Assistência e Proteção em Desastres', 'reconstrução em desastres' e 'recuperação pós-desastre'. Foram realizadas combinações entre esses termos em português e inglês, por meio do operador booleano $A N D$.

Nessa primeira fase, foi feita uma busca bibliográfica ampla, abarcando todos os tipos de desastre (não somente os relativos ao clima) e em todo o continente americano. $\mathrm{O}$ 'garimpo' de publicações que tratassem de desastres climáticos e que fossem de periódicos e/ou autores sul-americanos foi realizado posteriormente, em uma segunda fase (detalhada na figura 1). Isso se explica porque a intenção inicial era conhecer a situação global em situações de desastre nas Américas. No entanto, por conta do grande número de publicações levantadas, optou-se por restringir o escopo de análise e, por conseguinte, o objetivo da presente revisão. 
A segunda fase de busca, em que foi aplicado um segundo filtro, objetivou reunir apenas publicações que discorressem sobre desastres de origem geoclimática e de periódicos e/ou autores de países da América do Sul (publicações essas que não necessariamente discutissem desastres ocorridos em países sul-americanos). A intenção era conhecer o que autores e periódicos sul-americanos haviam publicado no período sobre a temática. Esse levantamento bibliográfico passou por três fases, detalhadas na figura 1.

Figura 1. Detalhamento do processo de busca e critérios de seleção da literatura

Fase 1: definição das bases de dados, dos descritores e definição dos critérios de inclusão e exclusão
Bases de dados: Portal BVS (Medline, Lilacs, Bireme) SciELO, Capes e Latindex.

Descritores: 'desastres', 'impacto psicossocial', 'apoio psicossocial', 'apoio social', 'saúde mental', 'capacidade de resposta ante emergência', 'efeitos de desastre na saúde', 'planejamento em desastres', 'socorro em desastres', 'Resgate, Assistência e Proteção em Desastres', 'reconstrução em desastres' e 'recuperação pós desastre'.

Panorama das Américas (periódicos e autores do continente americano)

Período: 1997 a 2016

Textos em português, inglês e espanhol

$\mathrm{N}=1.072+380 \mathrm{da}$ Latindex $=\mathbf{1 . 4 5 2}$

Critério de Inclusão: artigos científicos, teses, dissertações e manuais que abordassem os aspectos psicossociais em desastres. Publicações de 1996 a 2016, nos idiomas inglês, português e/ou espanhol e que tenham sido publicadas por periódicos de países do continente americano ou por autores desses países.

Critério de Exclusão: textos com foco na saúde animal em desastres (Medicina veterninária, Zootecnia etc.) e publicações cujos resumos não estivessem disponíveis para acesso.

Fase 2: filtragem das publicações
a partir do novo recorte (apenas
publicações que abordassem
desastres socioambientais de
origem geoclimática, de
periódicos e/ou autores da
América do Sul)

Fase 3: busca ativa dos textos na íntegra e resumos
Fora do tema desastres e aspectos psicossociais, fora do recorte temporal, geográfico (fora da América do Sul) e repetidos: $\mathrm{N}=1.190$ Indisponíveis: $\mathrm{N}=28$

Não abordavam desastres de origem geoclimática: $N=167$
48 (Medline, Lilacs, Bireme, SciELO e (apes) +4 (Ladindex)

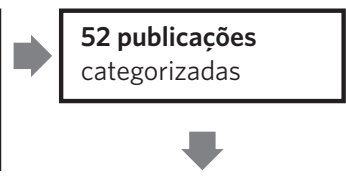

$\mathrm{N}=52$ (abordam, direta ou indiretamente, o tema dos aspectos psicossociais em desastres socioambientais de origem geoclimática)

34 abordam especificamente os desastres socioambientais de origem geoclimática

18 abordam pontualmente os desastres socioambientais de origem geoclimática - textos genéricos, que abordam desastres em geral, inclusive os geoclimáticos 
A busca inicial levantou 2.081 publicações apenas nas bases Capes e SciELO e no portal da BVS; e após a filtragem de materiais repetidos, fora do tema e do recorte temporal, totalizou 1.072 publicações. Na Latindex, não foi possível definir um recorte mais detalhado como o realizado nas outras bases indexadas, dados os limites de escopo da própria plataforma. Nesse caso, foi utilizado o termo 'desastres' como palavra de busca no filtro 'Diretório', uma das quatro bases de dados oferecidas pela Latindex. A opção por esse filtro se deu porque ele agrupa dados bibliográficos de todas as revistas registradas, tanto de publicações impressas como eletrônicas. A busca na Latindex localizou três revistas relacionadas com desastres, contudo, em apenas uma delas foram identificados materiais que se enquadravam no escopo da revisão: a revista Desastres y Sociedad (publicada por organismos internacionais e associada à Rede de Estudos Sociais em Prevenção de Desastres na América Latina, conhecida como La Red, situada no Panamá).

O levantamento contou com o recurso do Zotero, ferramenta de gerenciamento de bibliografias. Ele possibilitou a concentração em um só local e a organização de todas as publicações achadas nas buscas, com base nos descritores utilizados em cada base de dados e no total de materiais achados. Estes foram organizados por categorias temáticas, tipologia, periódico, país e ano de publicação, nacionalidade dos autores, objetivos das publicações e disponibilidade de acesso aos materiais. As publicações foram sistematizadas por categorias temáticas, considerando os temas dominantes e secundários dos materiais, a nacionalidade do(s) autor(es) e dos periódicos, ano de publicação e tipo de evento extremo.

\section{Resultados e discussão}

Os resultados indicaram uma produção de 52 textos ${ }^{11,14,15,24-72}$, no período de 20 anos, de autores e/ou periódicos sul-americanos, tendo em conta os critérios adotados nesta revisão. Esse número corresponde a uma média de 2,6 publicações por ano. Das 34 produções específicas sobre desastres socioambientais de origem geoclimática, 21 tinham como tema principal terremotos, mas tão somente os ocorridos no Chile e no Haiti em 2010. Também se destacaram as produções sobre enchentes e inundações (7) e seca (6), sendo que as relativas à seca se concentraram no ano de 2016 (4).

Os países com maior produção foram Brasil e Chile, como ilustra o gráfico 1: 
Gráfico 1. Distribuição das publicações da América do Sul no período de 1997 a 2016

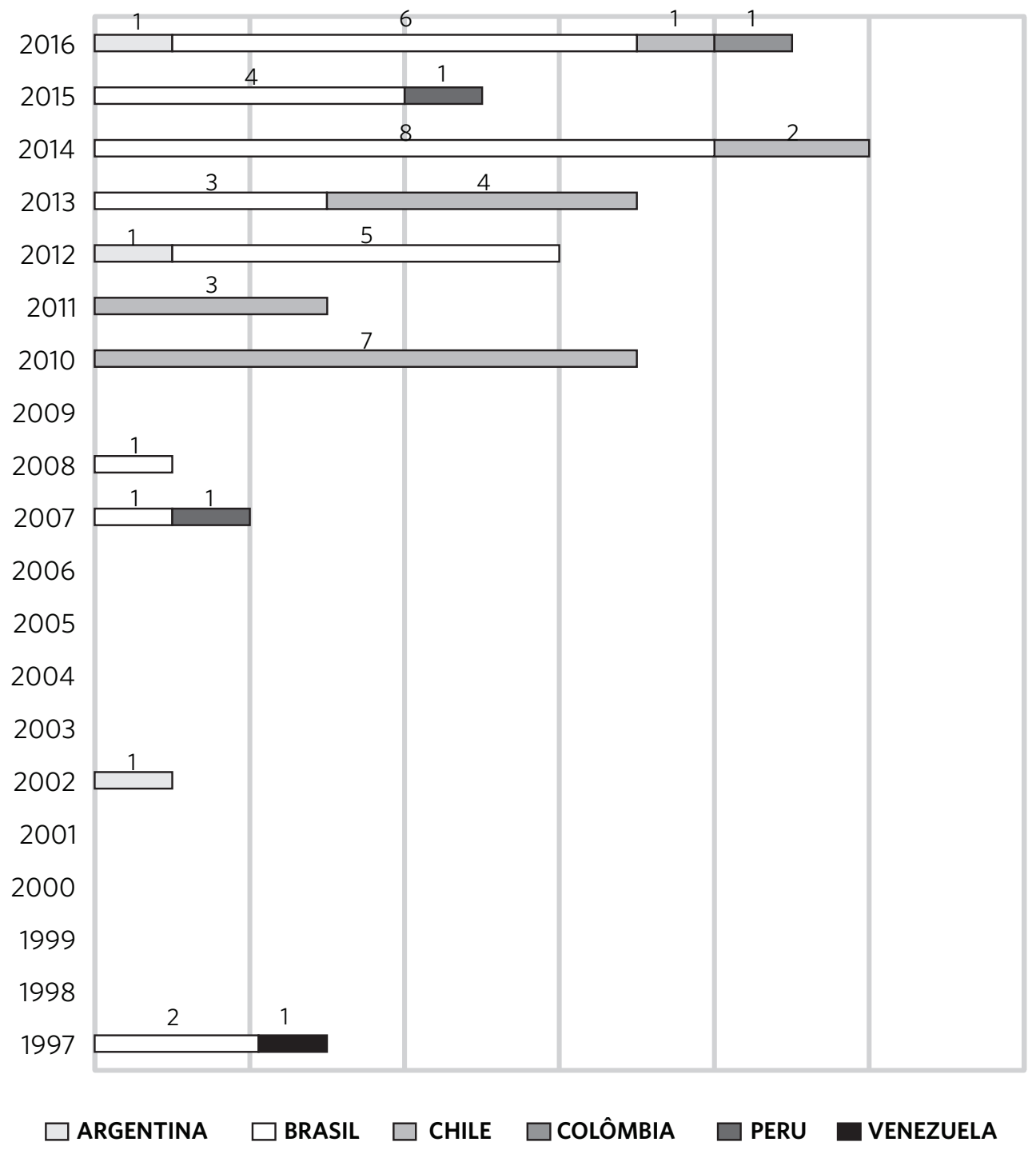

Fonte: Elaboração própria.

Os resultados também apontaram um aumento no número de publicações de autores e/ou periódicos da América do Sul a partir de 2010, com pico em 2014 e uma lacuna entre os anos de 1998 a 2001, de 2003 a 2006 e em 2009. Além disso, as publicações se concentraram em seis países da América do Sul, dos
12 que a integram: Brasil, Chile, Argentina, Peru, Venezuela e Colômbia.

Os aspectos psicossociais foram abordados nas publicações a partir de quatro temáticas principais: 'saúde mental', 'impactos psicossociais e na saúde', 'saúde pública' e 'gestão de risco', como pode ser visualizado no quadro 1 : 
Quadro 1. Temáticas mais discutidas nas publicações e discussões abordadas

\begin{tabular}{|c|c|}
\hline Temática & Enfoques \\
\hline $\begin{array}{l}\text { Saúde mental }(31)^{\mathbf{1 5}, \mathbf{2 4 -}} \\
\mathbf{4 4 , 5 2 , 5 5 , 6 3 , 6 4 , 6 6 - 6 8 , 7 1 , 7 2}\end{array}$ & $\begin{array}{l}\text { - Transtornos psicopatológicos (sintomatologia de TEPT - Transtorno de Estresse Pós- } \\
\text {-Traumático, depressão, ansiedade, estresse, transtornos adaptativos e Transtorno do } \\
\text { Deficit de Atenção com Hiperatividade - TDAH); Intervenção com sobreviventes (ên- } \\
\text { fase na fase de resposta ao desastre - manejo de crise); Experiências de intervenção } \\
\text { em Saúde mental após terremoto no Chile em } 2010 \text { (10); Importância da avaliação das } \\
\text { intervenções como estratégia de prevenção no desenvolvimento de patologias traumáti- } \\
\text { cas; Trabalho integrado com outros setores, articulado e contextualizado. }\end{array}$ \\
\hline $\begin{array}{l}\text { Impactos psicossociais e na } \\
\text { saúde }(38) \mathbf{1 1 , 1 4 , 1 5 , 2 4 , 2 6 , 2 9 - 3 8 , 4 5 -} \\
\mathbf{5 3 , 5 5 , 5 6 , 6 0 - 6 8 , 7 0 - 7 2}\end{array}$ & $\begin{array}{l}\text { - Mudança climática e os efeitos na saúde da população; Secas, inundações e enchentes } \\
\text { gerando desnutrição e impactos na saúde mental e ambiental; TEPT, distúrbios pós-trau- } \\
\text { máticos e depressivos, implicações psicossociais em moradores da zona rural de um } \\
\text { município do Nordeste; Vulnerabilidade socioambiental e grupos vulneráveis (crianças, } \\
\text { adolescentes e idosos) e abandono social. }\end{array}$ \\
\hline $\begin{array}{l}\text { Saúde pública (19)11,24- } \\
\mathbf{2 6 , 2 8 , 2 9 , 3 2 , 3 7 , 4 0 , 4 1 , 4 5 , 5 1 - 5 5 , 5 9 , 6 2 , 6 5}\end{array}$ & $\begin{array}{l}\text { - Associação entre os impactos dos desastres na saúde pública (estudos dos casos dos } \\
\text { estados de Santa Catarina em } 2008 \text { e Pernambuco em 2010, seca, enchentes, terremoto } \\
\text { e tsunami no Chile em 2010, enchentes e deslizamentos em Nova Friburgo em 2011); } \\
\text { Relação entre seca e saúde no Brasil, gerando desnutrição, impactos na saúde mental, } \\
\text { qualidade das águas e do ar, acesso aos serviços de saúde; Riscos sanitários em eventos } \\
\text { de massa e desastres; Biossegurança; Resposta do sistema de saúde e da cooperação } \\
\text { internacional no terremoto e tsunami de fevereiro de } 2010 \text { no Chile; Coordenação e } \\
\text { organização necessária dentro dos sistemas de saúde. }\end{array}$ \\
\hline $\begin{array}{l}\text { Gestão de risco } \\
(13) \mathbf{1 4 , 1 5 , 4 8 , 4 9 , 5 1 , 5 3 , 5 5 -} \\
\mathbf{5 7 , 6 2 , 6 9 , 7 0 , 7 2}\end{array}$ & $\begin{array}{l}\text { - Análise de risco como medida corretiva e preventiva, percepção de risco (hierarqui- } \\
\text { zação de riscos como parte do entendimento sobre a percepção de risco); Defesa Civil; } \\
\text { Análise da vulnerabilidade socioambiental e de grupos vulneráveis (vulnerabilidade } \\
\text { socioambiental criando condições para os desastres e limitando estratégias de preven- } \\
\text { ção e mitigação); Biossegurança (manejo de cadáveres, políticas públicas ineficientes, } \\
\text { degradação do meio, vulnerabilidade, mudança climática); Seca, estiagem, inundações } \\
\text { e terremoto no Haiti em 2010; Mudança climática, agenda de desenvolvimento susten- } \\
\text { tável, Rio+20; Risco associado a menor desenvolvimento socioeconômico; Produção de } \\
\text { sentidos (tensões entre gerenciamento de desastres socioambientais e a importância } \\
\text { do risco na vida cotidiana); Caracterização, indicadores e avaliação dos riscos de forma } \\
\text { integrada (humano e biota); Tecnologia de comunicação de risco como prática preventi- } \\
\text { va em saúde. }\end{array}$ \\
\hline
\end{tabular}

Fonte: Elaboração própria.

No período analisado (1997-2016), o ano com maior número de publicações foi 2014, com 10 produções. Um dado que se sobressaiu nos resultados desse ano foi o foco das produções ter sido os impactos psicossociais dos desastres sobre as populações, em estudos sobre vulnerabilidade e gestão de risco.

As publicações foram sistematizadas a partir de dois eixos temáticos estruturais: 'impactos psicossociais' e 'apoio psicossocial', como ilustra o quadro 2. 
Quadro 2. Eixos temáticos centrais, focos de análise identificados nas publicações e tipos de desastres mais comuns (identificados entre parênteses)

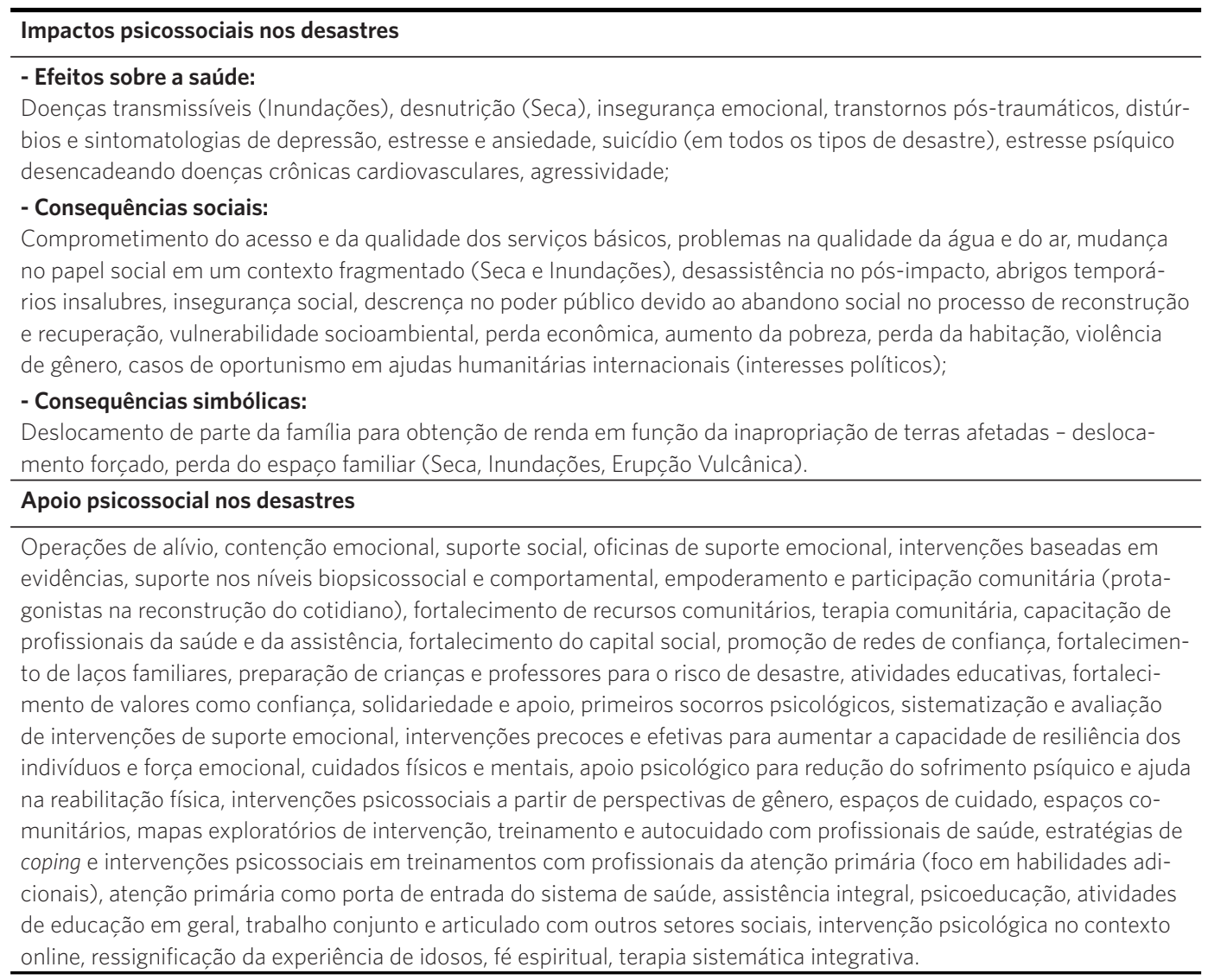
Fonte: Elaboração própria.

Quanto às metodologias utilizadas nas publicações, 21 foram pesquisas qualita-

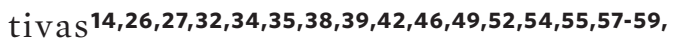
61,65,70,71 (com destaque para dois estudos de intervenção participativa $\left.{ }^{35,71}\right)$; 13 , quantitati$\operatorname{vas}^{25,29-31,37,47,48,50,60,62,66-69 ;}$; , revisões de literatura ${ }^{11,15,24,28,36,40,43-45,51,53,56,63,64,72}$ (sendo duas sistemáticas ${ }^{\mathbf{2 8 , 5 1}}$ ); e três estudos eram

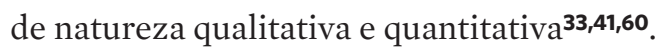

O período analisado nesta revisão foi marcado por importantes marcos estratégicos no que diz respeito à redução dos riscos de desastres no mundo. Podemos citar, inicialmente, a Estratégia Internacional para a Redução de Desastres (Eird/ISDR), adotada no ano 2000 pelos Estados-membros da ONU. A finalidade era reduzir as perdas geradas por desastres e construir comunidades e países resilientes para o enfrentamento desses eventos. $\mathrm{O}$ propósito era a sistematização das ações por cada país, a partir de plataformas nacionais para a redução de risco de desastres ${ }^{63}$. Além da Estratégia Internacional, pode-se citar o Marco de Ação de Hyogo ${ }^{73}$ (que estabeleceu metas a serem atingidas até o ano de 2015) e o Marco de Ação de Sendai74 (uma complementação do Marco de Hyogo, com ações definidas em 2015 e data-limite de 2030).

O Marco de Hyogo foi pactuado entre 168 países no ano de 2005 com o compromisso de desenvolver medidas de redução do risco de desastres. Tinha como objetivo estratégico 
alcançar, até o ano de 2015, uma redução dos riscos de desastres e aumentar a resiliência comunitária e das nações ${ }^{73}$. Portanto, a concentração de publicações do recorte temporal desta revisão no ano de 2014 pode ser explicada pela influência do Marco de Hyogo. Prestes a alcançar o ano limite da meta estabelecida para atingir os objetivos de redução de riscos de desastres do marco (2015), muitas produções se dedicaram, em 2014, aos impactos causados pelos desastres, na fase de resposta ao desastre e na questão da vulnerabilidade (grupos e ambientes vulneráveis).

Foram consideradas na sistematização deste artigo as cinco fases da Gestão de Riscos e Desastres (GRD) descritas na Política Nacional de Proteção e Defesa Civil75: prevenção, preparação, mitigação, resposta e recuperação.

Os anos de 2013, 2016 e 2010 tiveram o segundo maior número de publicações, com sete materiais cada. Acredita-se que as produções de 2016 tenham refletido a influência das discussões de outro marco, o de Sendai, adotado em 2015 pelos Estados-membros da ONU. Sem dúvida, esse importante instrumento, que complementou o Marco de Hyogo, mobilizou a comunidade científica a atuar de forma a reduzir os riscos e a ampliar as perspectivas de análise. Destaca-se que um dos aspectos-chave do Marco de Sendai foi a mudança de foco da redução de desastres para a redução de risco de desastres. Assim, o número de publicações em 2016 traduz, de certa forma, a preocupação com a mudança climática e com a agenda de desenvolvimento pós-2015.

No que concerne ao número significativo de materiais sobre seca no ano de 2016, é importante destacar o fenômeno El Niño em todo o globo. Muitos relatórios que tratam sobre o clima no planeta apontam que o ano de 2015 foi extremamente seco. Na América do Sul, por exemplo, apesar de muitos países da faixa subtropical terem apresentado clima úmido no ano de 2015, o Brasil aparece como tendo sido bastante afetado pela seca, com exceção do Sudeste ${ }^{6}$.

O predomínio das discussões sobre as fases de prevenção e resposta nos desastres e o pouco debate sobre a fase de recuperação/ reconstrução, que aparece em apenas sete publicações $\mathbf{2 6 , 3 0 , 5 3 , 5 8 , 6 1 , 6 3 , 6 5}$, evidenciam a necessidade não apenas de mais publicações na área, mas, sobretudo, a forte tendência ao enfoque na pronta resposta nesses contextos, revelando a adoção de estratégias pontuais e que não consideram em muitos casos os impactos em longo prazo nas diversas dimensões dos sujeitos. Quando explorada, a fase da recuperação vem associada à atuação do campo da saúde mental e aos impactos da intervenção tardia e/ou pontual na saúde psicológica em longo prazo, podendo levar a casos de Transtorno de Estresse Pós-Traumático (TEPT) e depressão.

Outro aspecto observado foi a ausência do termo 'psicossocial' em 13 publicações $\mathbf{3 4 , 3 8 , 4 0 , 4 1 , 4 3 , 5 0 , 5 2 , 5 4 , 5 7 , 6 0 , 6 2 , 6 4 , 7 2}$. Porém, mesmo não utilizando o termo, retrataram as dimensões psicológica e social em conjunto, pela perspectiva dos impactos nessas dimen-

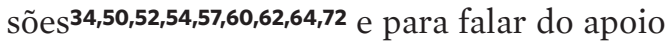
social e psicológico aos afetados $38,40,41,43$.

\section{Impactos psicossociais e na saúde}

Trinta e oito publicações exploraram, em alguma medida, os impactos dos desastres na dimensão psicossocial e na saúde, bem como o uso dos recursos psicossociais no enfrentamento ao desastre 11,14,15,24,26,29-38,45-53,55,56,60-68,70-72. A vulnerabilidade socioambiental, individual, estrutural ou institucional foi discutida em 11 publicações $\mathbf{1 4 , 1 5 , 2 4 , 2 6 , 4 5 , 4 6 , 4 9 , 5 0 , 5 6 , 7 0 , 7 2}$. Grupos vulneráveis, como idosos, crianças e adolescentes, tiveram destaque em sete delas ${ }^{32,33,35,46,66-68}$, em que os autores falaram da importância da psicoeducação na prevenção a danos evitáveis em nível social e emocional. Três desses estudos relataram a prevalência para TEPT em crianças e professores após o terremoto de 2010 no Chile66-68. Explicaram que as estratégias de psicoeducação, fortalecimento familiar e coesão social têm papel 
efetivo na redução dos impactos psicossociais nesses grupos vulneráveis. Expuseram os impactos do TEPT no desenvolvimento de crianças afetadas, com risco de apresentarem outras patologias em longo prazo $33,66-68$, e a necessidade de implementar programas preventivos para a população jovem afetada por desastres. Um dos estudos demonstrou que as políticas de alto impacto no enfrentamento aos desastres ao longo dos anos no Chile foram se adequando à realidade socioepidemiológica do País e que a saúde mental de crianças foi ganhando relevância ${ }^{32}$. Já o estudo sobre idosos chilenos tratou da ressignificação de redes e da própria vida, indicando que este também deve ser um grupo merecedor de atenção no planejamento em desastres ${ }^{46}$. Um aspecto interessante em algumas publicações que tratam dos impactos foi a perspectiva positiva do desastre. Alguns estudos definiram essas experiências potencialmente traumáticas como enriquecedoras, fazendo alusão à psicologia positiva e aos conceitos de resiliência e crescimento pós-traumático. Esses conceitos defendem a aprendizagem e as mudanças positivas diante de situações negativas e são considerados uma forma de adaptação a situações potencialmente traumáticas. Afirmam que há um componente capaz de trazer crescimento nesses contextos, e que deve ser explorado ${ }^{32,63,72}$. Em alguns estudos, a sobreposição de vulnerabilidades e impactos também foi investigada em decorrência do abandono social no processo de recuperação e reconstrução ${ }^{14,26}$.

\section{Saúde mental}

Trinta e uma publicações retrataram os aspectos psicossociais sob o enfoque da saúde mental15,2444,52,55,63,64,66-68,71,72. Falaram da importância de intervenções psicossociais precoces (nas primeiras horas que sucedem o dia do evento) para que não haja impactos em longo prazo na saúde mental, especialmente casos de TEPT e

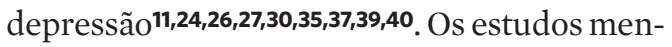
cionaram que a intervenção precoce atua na recuperação dos recursos psicossociais dos afetados ${ }^{64}$, que o apoio psicossocial ${ }^{27,29}$, mental e espiritual às vítimas, familiares e profissionais de saúde deve constar nos planos de ação ${ }^{51} \mathrm{e}$ que uma maior exposição nos desastres leva a maiores chances de desenvolver TEPT e distúrbios clinicamente significativos em longo prazo $26,52,55,67$. Um dado que chamou a atenção foi a mudança de paradigma quanto ao conceito de trauma, pois alguns estudos desta revisão (um deles pautado em revisões sistemáticas e meta-análises ${ }^{40}$ ) demonstraram a necessidade de intervenção psicológica precoce e contínua, levando em conta diversos setores e campos disciplinares ${ }^{30,63,68,40}$ e a importância do entendimento de que uma parcela dos afetados por desastre não necessariamente apresentará transtorno mental pós-traumático.

Desse modo, observa-se o deslocamento de uma visão patologizante e medicamentosa para uma abordagem que valoriza os aspectos psicossociais e o enfrentamento. Os primeiros cuidados psicológicos, a psicoeducação, a triagem psicológica nos primeiros dias após o evento, os trabalhos coordenados com a rede de saúde pública local e trabalho conjunto com assistentes sociais e profissionais da assistência humanitária são alguns exemplos de ações que reduzem os riscos do desenvolvimento de enfermidades pós-traumáticas. Nesse sentido, pode-se destacar a crítica feita, em uma das publicações, ao foco dado aos problemas de saúde mental na saúde pública ao longo dos anos, o que comprova que houve uma evolução na abordagem, dando lugar às discussões que privilegiam as ações psicossociais, a prevenção e a mitigação das consequências do desastre na saúde, e não somente os transtornos mentais ${ }^{36}$.

A perspectiva da psicologia positiva presente em algumas publicações $\mathbf{1 5 , 3 2 , 4 4 , 6 3 , 6 6 , 7 2}$ traz a ideia do desastre como evento que possibilita o crescimento e mobiliza recursos de enfrentamento. Os conceitos de resiliência e crescimento pós-traumático explicaram, por exemplo, o crescimento de políticas públicas e o fortalecimento da identidade nacional no Chile após o terremoto de 2010 segundo um 
dos estudos ${ }^{32}$. O desastre foi avaliado como uma oportunidade de crescimento para o País, marcado por inúmeros terremotos em sua história. Além desses aspectos, foi mencionado, em alguns estudos, o papel estratégico dos centros de saúde pública no monitoramento emocional dos afetados, que oferecem às pessoas os primeiros cuidados psicológicos, segurança, acolhimento, apoio social, informações e facilitam o retorno às suas atividades de rotina. Esses recursos são vistos como moderadores de risco para o desenvolvimento de possíveis patologias ${ }^{40}$.

O apoio social é citado como relevante fator psicossocial e gerador de maior satisfação na vida dos afetados, impactando sua saúde mental25. Nessa linha, um dos estudos identificou a correlação entre um baixo nível de apoio social e o aumento do sofrimento psicológico, indicando que, na cultura latino-americana, alguns dos elementos centrais de sua identidade são o coletivismo, a coesão social e a solidariedade, comparada a culturas individualistas ${ }^{72}$. O apoio psicossocial foi abordado com uma perspectiva não só de redução do sofrimento psíquico, mas também de reabilitação física ${ }^{30}$.

\section{Saúde pública}

A saúde pública foi investigada em $19 \mathrm{pu}-$ blicações ${ }^{11,24-26,28,29,32,37,40,41,45,51-55,59,62,65, ~}$ com enfoque no risco sanitário ${ }^{11,40,41,49,53}$, na importância do apoio mútuo entre os diversos órgãos que compõem o setor saúde de um país ${ }^{11,32,37,40,41,51}$, nos impactos na saúde e organização do setor nesses cená$\operatorname{rios}^{11,24,26,28,32,37,40,41,45,52-55,62}$, na participação comunitária como base para a intervenção psicossocial $24, \mathbf{4 1}, \mathbf{5 2}, \mathbf{5 9}, \mathbf{6 5}$, na atenção primária em saúde como elemento estratégi-

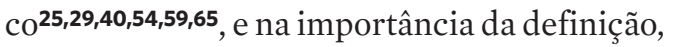
nas primeiras etapas da fase de resposta, dos processos psicossociais demandados nos desastres para apoiar vítimas, familiares e profissionais envolvidos $11,26,40,51,53,54$. Alguns estudos demonstraram dados de que uma maior exposição aos impactos de um desastre leva a distúrbios clinicamente significativos em longo prazo $26,52,55$, o que reforça a necessidade de o setor saúde estar atento à redução desses impactos e riscos em todas as fases do desastre. O monitoramento continuado e em longo prazo dos efeitos do desastre na saúde também foi recomendado"1, assim como o uso de práticas de intervenção psicossocial que têm apresentado resultados eficazes na saúde pública, como a terapia comunitária no Sistema Único de Saúde brasileiro (SUS) ${ }^{59} \mathrm{e}$ as experiências positivas do modelo de Saúde da Família no Chile ${ }^{54}$ e no Brasil65. Um dos enfoques dados ao risco sanitário foi debatido sob o prisma da saúde pública na publicação sobre biossegurança e manejo de corpos, associando a importância e rapidez desse serviço à redução da carga psicológica dos sobreviventes ${ }^{53}$.

\section{Gestão de risco}

Treze publicações trataram da gestão de risco (análise, percepção e comunicação de

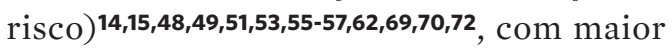
foco de análise na vulnerabilidade e na análise de riscos como prática preventiva. Um exemplo disso foi uma revisão realizada por autores chilenos sobre a percepção de riscos e desastres socioambientais de uma população potencialmente vulnerável no Chile ${ }^{72}$, em que apontaram a importância de obter informações sobre a percepção de risco para a formulação de campanhas educativas de prevenção em desastres, mostrando que o componente cultural também atravessa a forma com que os enfrentamos. Fizeram uma distinção interessante acerca das culturas individualistas e coletivistas, mencionando a cultura anglo-saxônica como exemplo de individualista e a latino-americana como coletivista, com maior predomínio de coesão cultural e solidariedade ${ }^{\mathbf{2}}$.

Ainda sobre a gestão de risco, as produções dos países da América do Sul refletem como estes têm lidado com os desastres: 
ainda se discute percepção de risco enquanto a tônica em outros locais do globo está bem mais avançada. Isso reforça a necessidade premente de políticas educativas, especialmente no Brasil.

Nesse sentido, a ausência de preocupação com as diversas dimensões do sofrimento social de grupos afetados por desastres foi identificada como ponto negativo em um estudo que avaliou a finalidade institucional da Defesa Civil no Brasil. Criticou-se seu aspecto tecnicista, que acaba não englobando dimensões importantes, como os impactos na fase de recuperação e as vulnerabilidades prévias ao evento ${ }^{\mathbf{1 4}}$.

No Chile, um estudo realizado para avaliar as atividades em saúde mental conduzidas pelos centros de atenção primária à saúde em Maule, região atingida pelo terremoto e pelo tsunami de 2010, indicou que houve dificuldade em lidar com os problemas de saúde mental desencadeados pelo desastre e que a não identificação das vulnerabilidades prévias do local interferiu na eficácia da assistência. A falta de coordenação, planejamento e exercícios locais também emergiu como problema para a efetividade das ações ${ }^{29}$.

\section{Mudança climática e as políticas de desenvolvimento sustentável}

Apenas cinco artigos da revisão referiam-se à mudança climática, à agenda política global pós-2015 e a políticas baseadas nos objetivos do desenvolvimento sustentável11,15,50,56,62, apesar das estratégias já citadas. É um número baixo ao considerar-se a importância global desse tema como eixo transversal das políticas. A seca foi o tema central de análise em três dessas publicações, ora relacionando-a com os riscos, hazards e os desastres, ora explorando a realidade do semiárido nordestino brasileiro e a tendência ao aumento da escassez de água até 2030 e seu impacto na saúde pública. Um artigo discorreu sobre as enchentes ${ }^{11}$, que também foram analisadas em relação aos seus reflexos na saúde pública. Os cinco artigos são de autores e periódicos brasileiros.

\section{Capital social e participação social como estratégias para o fortalecimento e a resiliência}

O capital social é definido como a capacidade de uma comunidade ou grupo para constituir diferentes tipos de associações, formais ou informais, permitindo o inter-relacionamento e a colaboração de seus participantes baseados nos princípios da confiança mútua, reciprocidade e cooperação ${ }^{60}$. Em um dos estudos realizados após o terremoto de 2010 no Chile, os autores sinalizaram, por meio de dados empíricos, que quando o capital social inicial de uma comunidade é muito baixo, o aumento da confiança comunitária, depois do desastre, é menor. Além disso, ressaltaram que o capital social baixo influi nos impactos do desastre, que aumentam em médio e longo prazo, e ainda favorece a violência no pós-desastre60.

O fortalecimento social também foi retratado sob a perspectiva das estratégias de adaptação, mostrando o efeito da fé espiritual como mediadora da angústia ligada à pobreza, esta última como uma dimensão psicossocial. Elaborou-se uma análise da relação entre o bem-estar psicossocial e a extrema pobreza, mostrando a realidade de uma favela no município de Fortaleza (CE), em que foi identificado um alto grau de percepção de bem-estar e de aspectos psicossociais causadores de ansiedade. Os autores apontaram as intervenções com terapia integrativa sistemática e o recurso da fé espiritual como 'âncoras de estabilidade emocional e bem-estar' e mostraram que há uma fragmentação nas abordagens científicas sobre as consequências psicossociais dos desastres em grupos em situação de pobreza e extrema pobreza. Indicaram o modelo de terapia integrativa sistemática como um valioso 'modificador de angústias' relacionadas a desastres e pobreza ${ }^{71}$.

A respeito do desastre na Região Serrana do estado do Rio de Janeiro, desencadeado por fortes chuvas e deslizamentos em janeiro de 2011, o levantamento revelou uma lacuna de produções que investigassem esse desastre 
tão emblemático ocorrido no Brasil: apenas quatro ${ }^{11,15,27,48}$. Vale frisar que esse desastre foi classificado pela ONU como o $8^{\circ}$ maior deslizamento de terra ocorrido no planeta nos últimos 100 anos, tendo sido comparado ao furacão Katrina de 2005 quando se trata de danos e magnitude ${ }^{\mathbf{1 2}}$. Dada a relevância desse acontecimento para o País, que salientou a desigualdade social e a vulnerabilidade socioeconômica e espacial dos locais onde houve deslocamentos de terra, a comunidade científica deveria ter se debruçado para analisar as iniciativas psicossociais realizadas nos locais afetados. Esse compartilhamento de experiências faz-se essencial para a multiplicação de práticas exitosas e é importante por representar práticas baseadas em evidências.

\section{Considerações finais}

Com o objetivo de contribuir para o conhecimento científico acerca dos desastres, esta revisão integrativa buscou responder 'como os aspectos psicossociais têm sido explorados na literatura científica nos últimos 20 anos (1997-2016) por autores e periódicos de países da América do Sul, em contextos de desastre socioambiental de origem geoclimática'. Considerando a importância dos aspectos psicossociais no contexto de desastres e seu papel no planejamento de ações, possibilitou um panorama sobre a produção sul-americana no período analisado, as diferentes perspectivas sobre os aspectos psicossociais em desastres, estratégias e abordagens utilizadas.

O que se observa é a necessidade de sair, particularmente o Brasil, de uma cultura do risco de desastres, que focaliza os eventos desencadeantes, para dar lugar a uma cultura de enfrentamento dos riscos a partir de suas causas estruturais, como a vulnerabilidade espacial e de grupos específicos. $\mathrm{O}$ foco na externalidade das causas dos desastres, ou seja, nos eventos físicos da natureza, torna-se uma perigosa forma de tentar compreendê-los e limita as ações de mitigação e prevenção. Observa-se uma atribuição do desastre a eventos físicos ameaçadores, e não aos processos sociais vigentes e ao uso inadequado do território.

No entanto, as publicações levantadas nesta revisão demonstraram a preocupação da comunidade científica sul-americana com as causas estruturais, cada vez mais relacionadas com as desigualdades sociais e vulnerabilidades socioambientais. De acordo com os estudos, os países da América do Sul precisam canalizar seus esforços para a construção de comunidades resilientes e para a minimização de problemas estruturais e de natureza social, política e econômica, como a diminuição das desigualdades e da vulnerabilidade social, sabidamente potencializadoras de desastres. No entanto, o que se vê, infelizmente, é uma produção que não se efetiva na prática; ações que estão condicionadas a decisões e interesses políticos, que minam os esforços para a redução de riscos de desastres.

Nota-se que a realidade do Chile, após os emblemáticos terremoto e tsunami de fevereiro de 2010 e de outros eventos semelhantes em seu território, 'moldou', como afirma um dos artigos, a construção da identidade e das políticas públicas nesse país, onde os processos de gestão de riscos de desastres foram sendo construídos ao longo dos anos com base na prevenção, na adaptação ao enfrentamento e em estratégias psicossociais calcadas na prevenção, resposta e recuperação, especialmente no rastreio e acompanhamento de sintomatologias psicopatológicas. No entanto, por falta de articulação com outros setores e de coordenação e planejamento, em alguns contextos, a população ficou desassistida e teve cobertura de saúde mental insuficiente.

Por fim, ressalta-se que, apesar do descritor 'psicossocial' e das palavras-chave contidas nos materiais, grande parte das publicações abordou exclusivamente o apoio e/ou os impactos psicológicos, desprezando outros elementos que a dimensão psicossocial engloba. Desse modo, esta revisão chama a atenção para o fato de que a maioria das publicações que contêm o termo 'psicossocial' 
ainda prioriza os aspectos psicológicos, mostrando forte tendência a focalizar o campo da saúde mental durante a análise dos aspectos 'psicossociais' em desastres. Contudo, o não reconhecimento das diversas dimensões do âmbito psicossocial em desastres pode gerar uma sobreposição de danos e atuar como fator gerador de outros desastres.

\section{Agradecimentos}

Ao apoio financeiro recebido da Coordenação de Aperfeiçoamento de Pessoal de Nível Superior - Brasil (Capes) e da Fundação Carlos
Chagas Filho de Amparo à Pesquisa do Estado do Rio de Janeiro (Faperj).

\section{Colaboradoras}

Carvalho MM (0000-0002-0433-0838)* contribuiu para a concepção, planejamento, análise e interpretação dos dados, elaboração do rascunho, revisão crítica do conteúdo e aprovação da versão final do manuscrito. Oliveira SS (0000-0001-5477-6216)* contribuiu para a concepção, planejamento, revisão crítica do conteúdo e aprovação da versão final do manuscrito.

\section{Referências}

1. Organizações das Nações Unidas Brasil. ONU: 90\% das mortes devido a desastres acontecem em países de renda baixa e média. [internet]. 27 de outubro de 2016 [acesso em 2017 out 10]. Disponível em: https://nacoesunidas.org/onu-90-das-mortes-devido-a-desastres-acontecem-em-paises-de-renda-baixa-e-media/.

2. Centre for Research on the Epidemiology of Disasters; The United Nations Office for Disaster Rick Reduction. The human cost of weather-related disasters 1995-2015 [internet]. 2015. [acesso em 2018 maio 15]. Disponível em: https://www.unisdr.org/files/46796 cop21weatherdisastersreport2015.pdf.

3. Organizações das Nações Unidas. The United Nations Office for Disaster Rick Reduction. Poverty and Death: Disaster Mortality 1996-2015 [internet]. [acesso em 2018 nov 4]. Disponível em: https://www.unisdr. org/we/inform/publications/50589.
4. Banco Mundial. Avaliação de perdas e danos: inundações e deslizamentos na Região Serrana do Rio de janeiro - janeiro de 2011. Relatório elaborado pelo Banco Mundial com apoio do Governo do Estado do Rio de Janeiro. Brasília, DF: Banco Mundial; 2012.

5. Brasil. Ministério da Saúde; Organização Pan-Americana da Saúde. Mudanças climáticas e ambientais e seus efeitos na saúde: cenários e incertezas para o Brasil. Brasília, DF: Organização Pan-Americana da Saúde; 2008.

6. World Meteorological Organization. The Global climate in 2013-2015, $\mathrm{N}^{\circ} .1179$ [internet]. 2016. [acesso em 2018 nov 16]. Disponível em: http://www.observatoriodoclima.eco.br/onu-liga-eventos-extremos-a-aquecimento/.

7. Field CB, Barros V, Stocker TF, et al., editores. IPCC 
2012: Managing the Risks of Extreme Events and Disasters to Advance Climate Change adaptation. A Special Report of Working Groups I and II of the Intergovernmental Panel on Climate Change. Cambridge: Cambridge University Press; 2012.

8. Pachauri RK. Reflections on COP 15. [internet]. 2010. [acesso em 2018 nov 18]. Disponível em: http:// www.fasid.or.jp/daigakuin/sien/kaisetsu/ doc_pdf/100108report.pdf.

9. Haines A, Kovats RS, Campbell-Lendrum D, et al. Climate change and human health: impacts, vulnerability and public health. Public Health. 2006; 120(7):585596.

10. Mata-Lima H, Alvino-Borba A, Pinheiro A, et al. Impactos dos desastres naturais nos sistemas ambiental e socioeconômico: o que faz a diferença? [internet]. Ambient. soc. 2013 [acesso em 2018 set 16]; 16(3):4564. Disponível em: https://www.scielo.br/pdf/asoc/ v16n3/v16n3a04.pdf.

11. Freitas CM, Ximenes EF. Enchentes e saúde pública: uma questão na literatura científica recente das causas, consequências e respostas para prevenção e mitigação. Ciênc. Saúde Colet. 2012; 17(6):1601-1616.

12. World Meteorological Organization. WMO. The Global Climate 2011-2015. [internet]. [acesso em 2018 abr 12]. Disponível em: https://public.wmo.int/en/ media/press-release/global-climate-2011-2015-hot-and-wild.

13. Alves ES, Francisco AL. Ação psicológica em saúde mental: uma abordagem psicossocial. Psicol. Ciênc. Profissão. 2009; 29(4):768-779.

14. Valencio NFLS. Disasters: technicism and social suffering. 2014; 19(9):3631-44.

15. Freitas CM, Carvalho ML, Ximenes EF, et al. Socio-environmental vulnerability, disaster risk-reduction and resilience-building: lessons from the earthquake in Haiti and torrential rains in the mountain range close to Rio de Janeiro in Brazil. Ciênc. Saúde Colet. [internet]. 2012; 17(6):1577-86.
16. World Bank \& United Nations. Natural hazards, unnatural disasters: the economics of effective prevention. Washington, DC: The International Bank for Reconstruction and Development; The World Bank; 2010.

17. United Nations Conference on Sustainable Development. Disaster-resilient Societies - Facts and figures. [internet]. 2012 [acesso em $2019 \mathrm{dez} 12$ ]. Disponível em: http://www.un.org/en/sustainablefuture/ disasters.shtml.

18. Narváez L, Lavell A, Ortega GP. La gestión del riesgo de desastres: un enfoque basado en procesos. San Isidro: Secretaría General de la Comunidad Andina; 2009.

19. Brasil. Ministério da Saúde. Desastres de origem natural [internet]. [acesso em 2018 out 26]. Disponível em: http://portalms.saude.gov.br/vigilancia-em-saude/vigilancia-ambiental/vigidesastres/desastres-de-origem-natural.

20. The Guardian. How climate change triggers earthquakes, tsunamis and volcanoes [internet]. 2016 out 16. The Guardian. [acesso em 2019 fev 10]. Disponível em: https://www.theguardian.com/world/2016/ oct/16/climate-change-triggers-earthquakes-tsunamis-volcanoes.

21. Liu C, Linde AT, Sacks IS. Slow earthquakes triggered by typhoons. Nature. 2009 [acesso em 2020 maio 7]; (459):833-836. Disponível em: https://doi. org/10.1038/nature08042.

22. Mcguire WJ. Waking the giant: how a changing climate triggers earthquakes, tsunamis, and volcanoes. Oxford: University Press; 2012.

23. Viterito A. The correlation of seismic activity and recent global warming. J earth sci clim change. 2016; (7):345.

24. Abeldaño RA, Fernández R. Salud mental en la comunidad en situaciones de desastre. Una revisión de los modelos de abordaje en la comunidad. Ciênc. Saúde Colet. 2016; 21(2):431-42. 
25. Portugal FB. Quality of life of primary care patients in Rio de Janeiro and São Paulo, Brasil: associations with stressful life events and mental health. 2016; 21(2):497-508.

26. Londe LR, Marchezini V, Conceição RS, et al. Impactos de desastres socioambientais em saúde pública: estudos dos casos dos estados de Santa Catarina em 2008 e Pernambuco em 2010. Rev bras estud popul. 2015; 32(3):537-62.

27. Weintraub ACA, Knobloch F, Vicente LN, et al. Atuação do psicólogo em situações de desastre: reflexões a partir da práxis. Interface comum. saúde educ. 2015; 19(53):287-98.

28. Silva JAM, Siegmund G, Bredemeier J. Crisis interventions in online psychological counseling. Trends in psyc. psychot. 2015; 37(4):171-82.

29. Vitriol V, Minoletti A, Alvarado R, et al. Respuesta de los centros de atención primaria en salud mental después del terremoto y tsunami del 2010 en la región del Maule. Rev med chil. 2014; 142(9):1120-7.

30. Guimaro MS, Steinman M, Kernkraut AM, et al. Psychological distress in survivors of the 2010 Haiti earthquake. Einstein (São Paulo). 2013; 11(1):11-4.

31. Guimaro MS. Post-traumatic stress disorder symptoms among professionals during humanitarian aid in Haiti after the earthquake in 2010. Ciênc. Saúde Colet. 2013; 18(11):3175-81.

32. Schonhaut BL. Terremotos, solidaridad y movilización nacional. Rev chil pediatr. 2013; 84(1):20-5.

33. Sommer AK, Barreau VM, Dávila DA. et al. Intervención de salud mental en niños expuestos a desastre natural. Rev chil pediatr. 2013; 84(1):59-67.

34. Gomes ERB, Cavalcante ACS. Desastres naturais: perdas e reações psicológicas de vítimas de enchente em Teresina-PI. Psicol soc. 2012; 24(3):720-8.

35. Correa A, Abufhele M, Briceño AM, et al. Intervención de salud mental para niños expuestos al desas- tre en la comunidad de Iloca: experiencia de la unidad de psiquiatría infantil de clínica alemana. Rev soc psiquiatr neurol infanc adolesc. 2011; 22(1):92-101.

36. Cova F, Rincón P. El terremoto y tsunami del 27 -f y sus efectos en la salud mental. Ter psicol. 2010; 28(2):17985.

37. Erazo C, Pemjean A. Red de atención de salud mental en chile y el terremoto de febrero 2010: fortalezas, daños y respuestas. Rev chil salud pública. 2010; 14(1):59-65.

38. García FE, Mardones R. Prevención de trastorno de estrés postraumático en supervivientes del terremoto de Chile de febrero de 2010: una propuesta de intervención narrativa. Ter psicol. 2010; 28(1):85-93.

39. Méndez MD, Leiva MC, Bustos CB, et al. Mapa exploratorio de intervenciones psicosociales frente al terremoto del 27 de febrero de 2010 en la zona centro-sur de Chile. Ter psicol. 2010; 28(2):193-202.

40. Figueroa RA, González M, Marín H. Apoyo psicológico en desastres: propuesta de un modelo de atención basado en revisiones sistemáticas y metaanálisis. Rev med chil. 2010; 138(2):143-51.

41. Loubat M, Fernández AM, Morales M. La experiencia de peralillo: una intervención psicológica para el estado de emergencia. Ter psicol. 2010; 28(2):203-7.

42. Peru. Ministerio de Salud. Dirección regional de salud ICA. Cómo trabajar salud mental comunitaria en situaciones de emergencia: lineamientos del IASC sobre salud mental y apoyo psicosocial en emergencias humanitarias y desastres. Lima: MS; 2007.

43. Comité permanente entre organismos. Guía del IASC sobre salud mental y apoyo psicosocial en situaciones de emergencia. Versión resumida. Ginebra: IASC; 2007.

44. Guimarães LAM, Guimarães PM, Neves SNH, et al. A técnica de debriefing psicológico em acidentes e desastres. Mudanças. 2007; 15(1):1-12. 
45. Benyakar M. Salud mental y desastres: nuevos desafíos. Revista neurol. Neuroc. y psiquiatría. 2002; 35(1):3-25.

46. Alpino TA. Disasters related to droughts and public health - a review of the scientific literature. Ciênc. Saúde Colet. 2016; 21(3):809-20.

47. Díaz CA, Lamig MAV, Venegas MQ, et al. Re-signification of life experience in older adults affected by three kinds of natural disasters in Chile. FQS. 2016; 17(1):1-37.

48. Camurça CE, Alencar A, Cidade E, et al. Implicações psicossociais da seca na vida de moradores de um município da zona rural do nordeste do Brasil. Avanc. psicol. Latino-americana. 2016; 34(1):117-128.

49. Freitas CM, Silva DRX, Sena ARM, et al. Natural disasters and health: an analysis of the situation in Brazil. Ciênc. Saúde Colet. 2014; 19(9):3645.

50. Spink MJP. Viver em áreas de risco: tensões entre gestão de desastres ambientais e os sentidos de risco no cotidiano. Ciênc. Saúde Colet. 2014; 19(9):3743-54.

51. Xavier D, Barcellos C, Freitas C. Extreme weather events and their consequences on health: the 2008 disaster in Santa Catarina taking into account different information sources. Ambiet. soc. [internet]. 2014; 17(4):273-290.

52. Castro CF, Simoes DCM, Delamarque EV, et al. Mass events, disasters and public health/Eventos de massa, desastres e saúde pública. Ciênc. Saúde Colet. 2014; 19(9):3717.

53. Shoaf K. Organizing the health sector for response to disasters. Cad. Saúde Pública. 2014; 19(9):3705-15.

54. Cardoso TAO. Biosecurity and disaster: concepts, prevention, public health and management of corpses. Physis. 2012; 22(4):1523-42.

55. López TE, Santana NP. El terremoto de 2010 en chile: respuesta del sistema de salud y de la cooperación internacional. Rev panam salud pública. 2011; 30(2)160-6.
56. Grigoletto JC, Cabral AR, Bonfim CV, et al. Management of health sector actions in drought situations. Ciênc. Saúde Colet. 2016; 21(3):709.

57. Favero E, Diesel V. A seca enquanto um hazard e um desastre: uma revisão teórica. Aletheia. 2008; (27):198.

58. Bodstein AV. The vulnerability of the elderly in disasters: the need for an effective resilience policy. Ambient. Soc. 2014; 17(2):171-185.

59. Cueto RM, Fernández MZ, Moll S, et al. Community participation and strengthening in a reconstruction context after a natural disaster. J prev interv community. 2015; 43(4):291-303.

60. Santos Padilha C, Oliveira WF. Community therapy: practice reported by professionals within SUS in Santa Catarina, Brazil. Rev Gaúcha Enfer. 2012; 16(43):1069.

61. Dussaillant F, Guzmán E. Trust via disasters: the case of Chile's 2010 earthquake. Disasters. 2014; 38(4):80832 .

62. Magaña Frade I, Silva-Nadales S, Rovira Rubio R. Catástrofe, subjetividad femenina y reconstrucción: aportes y desafíos desde un enfoque de género para la intervención psicosocial en comunidades afectadas por el terremoto. Ter Psicol. 2010; 28(2):169-77.

63. Sena A, Barcellos C, Ramalho W, et al. Measuring the invisible: analysis of the sustainable development goals in relation to populations exposed to drought. Ciênc. Saúde Colet. 2016; 21(3):671-84.

64. Paranhos ME, Werlang BG. Psicologia nas emergências: uma nova prática a ser discutida. Psicol. cienc. prof. 2015; 35(2):557-71.

65. Rocha V, Freitas CM. The theme of disaster in health care: profile of technical and scientific production in the specialized database on disasters of the virtual health library - vhl. Ciênc. Saúde Colet. 2014; 19(9):3775-90.

66. Fernandes GCM, Boehs AE, Heidemann ITSB. O su- 
porte social durante a transição familiar no pós-desastre natural. Texto \& contexto - Enfermagem. 2013; 22(4):1098-105.

67. Cova F, Valdivia M, Rincón P, et al. Estrés postraumático en población infantojuvenil post $27 \mathrm{f}$. Post-traumatic stress disorder in young population after the natural disaster on february 27, 2010. Rev soc psiquiatr neurol infanc adolesc. 2011; 22(1):2-11.

68. Briceño AM, Abufhele M, Dávila AM, et al. Estrés postraumático en escolares a 8 meses del 27f. Post-traumatic stress disorder in children in a coastal town eight months after the earthquake/tsunami. Rev soc psiquiatr neurol infanc adolesc. 2011; 22(1):12-20.

69. Bedregal P, Carvallo C, Hernández V, et al. Estrés post-traumático post terremoto $27 \mathrm{f}$ en cuidadores principales de niños preescolares, factores asociados del cuidador, de la familia y la crianza. Post-traumatic stress disorder after 27/f earthquake in primary caregivers of preschool children, factors associated with the caregiver, family and parenting. Rev soc psiquiatr neurol infanc adolesc. 2011; 22(1):21-28.

70. Rojas MC, Meichtry NC, Vázquez JC, et al. El riesgo de la vivienda urbana para la salud de la población. Un análisis desde la salud colectiva y la vulnerabilidad social. 2012.

71. Panza R, Wiesenfeld E. Las tres caras de los desastres: percepción de riesgo, derrumbe y reubicación. Rev. Desast. socied. [internet]. 1997 [acesso em 2017 mar 15]; 5(8):76-90. Disponível em: https://www.desenredando.org/public/revistas/dys/.
72. Mocellin J, Barreto A, Gural D. Pobreza y desastres: terapia integrante sistemática en una barriada pobre de Brasil Rev. Desast. socied. [internet]. 1997 [acesso em 2017 mar 15]; 5(8):69-77. Disponível em: https:// www.desenredando.org/public/revistas/dys/.

73. Díaz EM, Jarau PO. Psicología y desastres ambientales en Chile. R Rev. Desast. socied. [internet]. 1997 [acesso em 2017 mar 15]; 5(8):78-95. Disponível em: https://www.desenredando.org/public/revistas/dys/.

74. United Nations International Strategy for Disaster Reduction. Hyogo framework for action 2005-2015: building the resilience of nations and communities disasters [internet]. 2005 [acesso em 2018 jul 8]. Disponível em: https://www.unisdr.org/.

75. United Nations International Strategy for Disaster Reduction. UNISDR. Sendai framework for disaster risk reduction 2015-2030. [internet]. 2015. [acesso em 2018 jul 8]. Disponível em: http://www.wcdrr. org/uploads/Sendai_Framework_for_Disaster_Risk_ Reduction_2015-2030.pdf.

76. Brasil. Lei ${ }^{\circ} 12.608$, de 10 de abril de 2012. Política Nacional de Proteção e Defesa Civil. Diário Oficial da União. 11 Abr 2012.

Recebido em 29/09/2019
Aprovado em 10/01/2020

Conflito de interesses: inexistente

Suporte financeiro: Coordenação de Aperfeiçoamento de Pessoal de Nível Superior - Brasil (Capes) - Código de Financiamento 001 - e Fundação Carlos Chagas Filho de Amparo à Pesquisa do Estado do Rio de Janeiro (Faperj) - Programa Bolsa Nota 10 -

Doutorado 